\title{
Anti-histamínicos no resfriado comum: uma prescrição igualmente comum
}

José Agostinho Santos*

\section{RESUMO}

Objectivos: A literatura salienta a popularidade, entre a comunidade médica, da abordagem terapêutica com anti-histamínicos para o resfriado comum. $O$ objectivo deste trabalho consiste em rever a evidência sobre a efectividade e a segurança dos anti-histamínicos em monoterapia no resfriado comum em pacientes pediátricos e adultos.

Fontes de dados: Medline, sítios de medicina baseada na evidência, Índex de Revistas Médicas Portuguesas e referências bibliográficas dos artigos seleccionados.

Métodos de revisão: Pesquisa de normas de orientação clínica (NOC), revisões sistemáticas e ensaios clínicos aleatorizados e controlados (ECAC), publicados entre Janeiro/1980 e Outubro/2010, utilizando os termos MeSH: antihistamines e common cold. Foi utilizada a escala Strength Of Recommendation Taxonomy (SORT) para atribuição dos níveis de evidência e forças de recomendação.

Resultados: Foram encontrados 70 artigos, dos quais sete foram seleccionados: duas NOC para abordagem do resfriado comum que não incluem anti-histamínicos em monoterapia como opção terapêutica; uma meta-análise da Cochrane que não encontrou benefício clínico com o uso de anti-histamínicos em monoterapia e que sugere um risco acrescido de efeitos sedativos com os de 1. a geração. Os autores recomendam a descontinuação da prescrição destes fármacos no resfriado comum (nível de evidência 1); quatro ECAC de boa qualidade nos quais se verificou que a terapêutica com diferentes anti-histamínicos foi tão eficaz quanto o placebo na redução da tosse aguda e, em três dos quais não se apresentaram diferenças significativas nos efeitos laterais (nível de evidência 1).

Conclusões: A evidência disponível indica que o uso de anti-histamínicos em monoterapia na terapêutica sintomática do resfriado comum não é efectivo em crianças e adultos (SOR A). Diversos estudos sugerem um risco acrescido de efeitos sedativos com esta terapêutica (SOR B). Esta sedação é frequentemente confundida com uma prostração que sugeriria um agravamento do quadro, algo a acrescentar a um custo desnecessário associado a esta prescrição que apenas é evidentemente comum.

Palavras chave: Anti-histamínicos; Resfriado Comum.

\section{INTRODUÇÃO}

A s infecções agudas das vias respiratórias superiores são o segundo diagnóstico mais frequente no gabinete médico e o resfriado comum constitui o síndrome vírico mais prevalente. ${ }^{1}$ Este afecta mais as crianças (seis vezes/ano) do que os adultos (quatro vezes/ano). 30-35\% dos resfriados comuns são causados pelos mais de 100 tipos do rinovírus. Outros vírus envolvidos são o coronavirus, vírus sincicial respiratório, vírus parainfluenza e adenovirus. $^{2}$

\footnotetext{
* Interno de Medicina Geral e Familiar, USF Lagoa, Centro de Saúde da Senhora da Hora, ULS - Matosinhos.
}

Tipicamente, o resfriado comum começa por uma fase prodrómica com sintomas como mau-estar inespecífico, odinofagia, mialgias e febrícula. É frequentemente a partir do terceiro dia de evolução que o resfriado adquire o quadro perturbador para o paciente com o surgimento de rinorreia, congestão nasal, espirros e tosse, conduzindo-o, muitas vezes, à abstinência laboral e ao recurso aos Serviços de Saúde. ${ }^{3}$

A sua terapêutica em ambulatório é puramente sintomática. ${ }^{1,2}$ Com o suposto intuito de alívio dos sintomas, existem diversos medicamentos não-sujeitos a receita médica, que estão vulgarizados entre a população geral. ${ }^{4}$ Nos EUA, 25\% dos adultos ingeriu um fármaco, 
prescrito ou não-prescrito, para controlo de sintomas do resfriado comum na semana anterior. ${ }^{1} \mathrm{~A}$ literatura revela que, por sua vez, os anti-histamínicos (AH) se encontram muito popularizados entre a comunidade médica, principalmente na hora da prescrição. ${ }^{5}$

A eficácia dos anti-histamínicos na rinite alérgica está comprovada, com boa relação benefício/custo. A sua acção na rinite vírica é questionável e tem sido suportada por relatos pouco claros de benefício e com víeses de confundimento, mas que têm sido suficientes para perpetuar a sua prescrição perante um resfriado comum. ${ }^{6}$

Assim, o objectivo deste trabalho consistiu em rever a evidência disponível sobre a efectividade (ou melhoria sintomática) e a segurança (ou efeitos laterais) do uso de anti-histamínicos em monoterapia no resfriado comum.

\section{MÉTODOS}

Foi realizada uma pesquisa de normas de orientação clínica (NOC), revisões sistemáticas com ou sem metaanálises e ensaios clínicos aleatorizados e controlados (ECAC), nas fontes de dados National Guideline Clearinghouse, Guidelines Finder, Canadian Medical Association Practice Guidelines, Cochrane, DARE, Bandolier, Medline, Índex de Revistas Médicas Portuguesas e referências bibliográficas dos artigos seleccionados, publicados entre Janeiro de 1980 e Outubro de 2010, nas línguas portuguesa, inglesa e espanhola e utilizando as palavras-chave (termos MeSH): antihistamines e common cold.

O critérios de inclusão dos artigos consistiram numa população constituída por indivíduos com resfriado comum, cuja intervenção fosse o uso de anti-histamínicos em monoterapia comparativamente ao uso de placebo ou à ausência de qualquer terapêutica farmacológica, e cujos resultados medidos fossem a variação sintomatológica (estado global e/ou variação da quantidade ou duração da rinorreia, da congestão nasal, dos espirros ou da tosse aguda) e os efeitos laterais.

Foram excluídos os estudos que incluíam administração concomitante de outros fármacos ou indivíduos com patologias com impacto potencialmente enganador na medição dos resultados: eczema atópico, rinite alérgica, asma, doença crónica respiratória e doença respiratória com sobreinfecção bacteriana.
Foi utilizada a escala Strength Of Recommendation Taxonomy (SORT) da American Academy of Family Physicians $^{7}$ para avaliação da qualidade dos estudos e atribuição das forças de recomendação.

\section{RESULTADOS}

Foram encontrados 70 artigos, sete dos quais foram seleccionados: duas Normas de Orientação Clínica (NOC), uma meta-análise e quatro ensaios clínicos aleatorizados controlados.

A NOC do American College of Chest Physicians é baseada em evidência clínica e foi publicada em $2006 .{ }^{8} \mathrm{O}$ Colégio Americano documenta, com uma força de recomendação A (após conversão para a SORT), que os AH não-sedativos em monoterapia são ineficazes para redução da tosse aguda e que não devem ser prescritos em contexto de resfriado comum. Salienta que alguns estudos revelaram que a associação de $\mathrm{AH}$ de $1 .^{a}$ geração com anti-inflamatório não-esteróide (AINE) ou com fármaco descongestionante poderia ser eficaz na redução da tosse aguda. Porém, estabelece que estes AH sedativos não devem ser usados em monoterapia.

A NOC para o diagnóstico e tratamento das doenças respiratórias em crianças e adultos do Institute of Clinical Systems Improvement foi elaborada baseando-se em evidência e data de 2008. ${ }^{9}$ Entre as suas linhas orientadoras para a terapêutica sintomática do resfriado comum, não faz qualquer referência ao uso de $\mathrm{AH}$ em monoterapia.

A meta-análise encontrada (Quadro I) é da Cochra$n e$, de 2003, e incluiu 22 ECAC, com um total de 7554 pacientes com resfriado comum e com idades $\geq 6$ anos e 150 pacientes com $<6$ anos. ${ }^{2}$ A intervenção estudada foi o uso de diferentes $\mathrm{AH}$ sedativos e não-sedativos em monoterapia para os sintomas do resfriado comum (estado geral, congestão nasal, rinorreia e espirros) num grupo comparativamente ao placebo ou ausência de tratamento farmacológico no grupo controlo. Os resultados desta meta-análise integraram diversas e complexas comparações para um mesmo sintoma e revelaram que, quanto a eficácia, os AH não têm benefício clínico global (definido pelos autores da meta-análise como uma diferença maior de $15 \%$ de individuos que referiam melhoria sintomática relevante entre os dois grupos). Quanto a segurança, o uso de AH de 1. ${ }^{\mathrm{a}}$ geração associou-se a maiores efeitos sedativos e gastroin- 


\begin{tabular}{|c|c|c|c|c|}
\hline Referência & População & Intervenção & Resultados & $\begin{array}{l}\text { Nível de } \\
\text { evidência }\end{array}$ \\
\hline $\begin{array}{l}\text { Sutter et al, } 2003 \\
\text { Cochrane Reviews } \\
22 \text { ECAC }\end{array}$ & $\begin{array}{l}n=7554 \text { com } \\
\text { idade } \geq 6 \text { anos }+ \\
150 \text { com }<6 \text { anos } \\
\text { Resfriado comum }\end{array}$ & $\begin{array}{l}\text { Anti-histamínico } \\
\text { (terfenadina, clorfeniramina, } \\
\text { cetirizina, triprolidina, clemastina, } \\
\text { dexbronfeniramina, doxilamina, } \\
\text { bronfeniramina, loratadina, } \\
\text { azatadina, benadril, fenidramina, } \\
\text { tripelenamina, tonzilamina, } \\
\text { difenidramina) } \\
\text { vs } \\
\text { Placebo ou ausência de } \\
\text { tratamento farmacológico }\end{array}$ & $\begin{array}{l}\text { Efectividade: } \\
\text { Anti-histamínico sem benefício } \\
\text { clínico global (diferença entre } \\
\text { grupos < } 15 \% \text { ) } \\
\text { Segurança (Sedação e } \\
\text { sintomas GI) } \\
\text { Anti-histamínico de 1. }{ }^{\text {a geração }} \\
\text { > placebo* } \\
\text { Sem tradução clínica }\end{array}$ & 1 \\
\hline
\end{tabular}

* diferença estatisticamente significativa

testinais, porém os autores desta meta-análise consideraram que tal diferença, apesar de estatisticamente significativa, não teria importante tradução clínica. Estes mesmos autores recomendam que o uso de anti-histamínicos deve ser descontinuado para o tratamento do resfriado comum (nível de evidência 1).

Os quatro ECAC seleccionados (Quadro II) estudaram o uso de AHs na tosse aguda associada ao síndrome vírico, sintoma não avaliado na meta-análise anterior.

O primeiro ECAC seleccionado é da autoria de Gaffey et al. ${ }^{6}$ Trata-se de um estudo que preenche os critérios de boa qualidade: dupla-ocultação assegurada, uma boa dimensão amostral e um follow- $u p \geq 80 \%$. Este ensaio incluiu 250 pacientes adultos com resfriado comum, a metade dos quais foi administrado o $\mathrm{AH}$ nãosedativo terfenadina $60 \mathrm{mg} 2 \mathrm{x} /$ dia durante 3,5 dias e placebo no mesmo esquema posológico à outra metade. Os resultados (medidos por uma escala subjectiva de preenchimento pelo doente) não revelaram diferenças estatisticamente significativas na eficácia em reduzir a tosse aguda ou na segurança entre os dois grupos. Tratando-se de um estudo consistente, foi-lhe atribuído um nível de evidência 1.

Berkowitz e colegas realizaram, em 1991, um ECAC envolvendo 100 adultos com resfriado comum, usando o mesmo anti-histamínico terfenadina por 5 dias com o dobro da dose e comparando também com pla- cebo. ${ }^{10}$ Teve dupla-ocultação, boa dimensão amostral e bom follow-up. Os resultados não revelaram diferenças estatisticamente significativas quer a nível de eficácia na redução da tosse aguda, quer a nível da segurança entre os dois grupos (nível de evidência 1).

O ECAC conduzido por Sakchainanont et al, de 1990, incluiu 143 pacientes pediátricos com idades $<5$ anos e com resfriado comum, a um terço dos quais foi administrado o AH sedativo clemastina $2 x$ /dia, a outro terço o AH sedativo clorfeniramina $3 \mathrm{x} /$ dia e ao último terço placebo $2 \mathrm{x} /$ dia, durante 3 dias. ${ }^{11}$ Os resultados não revelaram diferenças estatisticamente significativas na eficácia na redução da tosse aguda ou na incidência de efeitos laterais entre os três grupos.

Finalmente, Paul et al elaboraram um ECAC em 2004, que integrou 100 pacientes pediátricos, um terço dos quais foi submetido a uma toma única nocturna de AH de 1. ${ }^{a}$ geração difenidramina e noutro terço foi usado placebo no mesmo esquema. ${ }^{12}$ Um terceiro grupo foi independentemente submetido a toma única nocturna de dextrometorfano, cujos resultados escapam ao âmbito desta revisão. Os resultados medidos na manhã seguinte, segundo um formulário elaborado aos pais questionando sobre a qualidade e a quantidade do sono do doente e dos pais e sobre a frequência e severidade da tosse das crianças, não encontraram diferenças estatisticamente significativas na eficácia na redução da 


\begin{tabular}{|c|c|c|c|c|}
\hline Referência & População & Intervenção & Resultados & $\begin{array}{l}\text { Nível de } \\
\text { evidência }\end{array}$ \\
\hline $\begin{array}{l}\text { Gaffey et al, } \\
1988\end{array}$ & $\begin{array}{l}\text { EUA } \\
\mathrm{n}=250 \\
\text { (média = } 23 \text { anos) } \\
\text { Resfriado comum }\end{array}$ & $\begin{array}{l}\text { Terfenadina } 60 \mathrm{mg} \\
\text { vs } \\
\text { Placebo } \\
2 \text { vezes/dia, 3,5 dias }\end{array}$ & $\begin{array}{l}\text { Efectividade na tosse aguda: } \\
\text { Terfenadina } \approx \text { Placebo } \\
\text { Efectividade global ( } 6 \text { sintomas, } \\
\text { incluindo tosse): } \\
2,2 \pm 1,1 \text { vs } 2,1 \pm 1,3 \\
\text { Efeitos laterais: } \\
\text { Fadiga } \\
12 \% \text { vs } 10 \%\end{array}$ & 1 \\
\hline $\begin{array}{l}\text { Berkowitz et al, } \\
1991\end{array}$ & $\begin{array}{l}\text { EUA } \\
\mathrm{n}=100 \\
\text { (média = } 32 \text { anos) } \\
\text { Resfriado comum }\end{array}$ & $\begin{array}{l}\text { Terfenadina } 120 \mathrm{mg} \\
\text { vs } \\
\text { Placebo } \\
2 \text { vezes/dia, } 5 \text { dias }\end{array}$ & $\begin{array}{l}\text { Efectividade na tosse aguda: } \\
\text { Terfenadina } \approx \text { Placebo } \\
0,81 \pm 0,13 \text { vs } 0,65 \pm 0,12 \\
\text { Efeitos laterais: } \\
\text { Cefaleias } \\
6,1 \% \text { vs } 4,0 \%\end{array}$ & 1 \\
\hline $\begin{array}{l}\text { Sakchainamont } \\
\text { et al, } \\
1990\end{array}$ & $\begin{array}{l}\text { Tailândia } \\
\mathrm{n}=143 \\
\text { (média = } 23 \text { meses) } \\
\text { Resfriado comum }\end{array}$ & $\begin{array}{l}\text { Clemastina } \\
0,05 \mathrm{mg} / \mathrm{kg} / \text { dia } 2 x / \text { dia } \\
\text { vs } \\
\text { Clorfeniramina } \\
0,35 \mathrm{mg} / \mathrm{kg} / \text { dia } 3 x / \text { dia } \\
\text { vs } \\
\text { Placebo } 2 x / \text { dia } \\
3 \text { dias }\end{array}$ & $\begin{array}{l}\text { Efectividade na tosse aguda: } \\
\text { Clemastina } \approx \text { Clorfeniramina } \approx \text { Placebo } \\
39,6 \% \text { vs } 39,6 \% \text { vs } 27,6 \% \\
\text { Efeitos laterais: } \\
\text { Sonolência/Sedação } \\
\text { Clemastina } \approx \text { Clorfeniramina } \approx \text { Placebo }\end{array}$ & 1 \\
\hline $\begin{array}{l}\text { Paul et al, } \\
2004\end{array}$ & $\begin{array}{l}\text { EUA } \\
\mathrm{n}=100 \\
\text { (média }=4,5 \text { anos) } \\
\text { Resfriado comum }\end{array}$ & $\begin{array}{l}\text { Difenidramina } 1,25 \mathrm{mg} / \mathrm{kg} \\
\text { vs } \\
\text { Placebo } \\
\text { (vs dextrometorfano) } \\
\text { Toma única nocturna }\end{array}$ & $\begin{array}{l}\text { Efectividade na tosse aguda } \\
\text { (diferença da pontuação no } \\
\text { questionário aplicado aos pais): } \\
\text { Difenidramina } \approx \text { Placebo } \\
11,8 \text { vs } 10,8 \text { (vs } 10,8 \text { ) } \\
\text { Efeitos laterais: } \\
\text { Sonolência/Sedação } \\
3 / 33 \text { vs } 0 / 33^{*} \text { (vs } 0 / 33 \text { ) }\end{array}$ & 1 \\
\hline
\end{tabular}

* diferença estatisticamente significativa

tosse aguda. A sonolência/sedação foi o efeito lateral mais referido no grupo que fez AH sedativo comparativamente ao grupo placebo, uma diferença que foi estatisticamente significativa.

Estes dois últimos ECAC, envolvendo uma popula- ção pediátrica, apresentaram boa amostragem, foram duplamente cegos com atribuição oculta, tiveram um seguimento superior a $80 \%$ e um resultado orientado para o paciente, pelo que lhes é atribuído um nível de evidência 1. 


\section{CONCLUSÕES}

A evidência relativa a esta temática é considerável. Quanto à efectividade dos AHs nos sintomas do resfriado comum, uma meta-análise consistente da Cochrane apresenta como principal conclusão que a prescrição dos AHs em monoterapia deve ser descontinuada no resfriado comum em idade adulta ou pediátrica. Dois ECAC envolvendo uma população pediátrica e outros dois envolvendo população adulta - os quatros de boa qualidade metodológica - documentaram efeitos semelhantes entre o AH e o placebo na redução da tosse aguda associada ao síndrome vírico.

Assim, o uso de anti-histamínicos em monoterapia na terapêutica sintomática do resfriado comum não apresenta efectividade clínica (SOR A), recomendação que vai de encontro às normas de orientação clínica elaboradas pelas sociedades científicas internacionais.

Quanto à segurança, a mesma meta-análise da Cochrane documenta uma maior incidência de efeitos sedativos e gastrointestinais com o uso de AH de 1. ${ }^{a}$ geração comparativamente ao placebo, diferença que foi estatisticamente significativa nalguns ensaios incluídos embora clinicamente pouco relevante. Os resultados dos ECAC que utilizaram anti-histamínicos sedativos não expuseram tal associação.

Uma vez que nem todos os estudos parecem ser concordantes, o uso de anti-histamínicos de 1. 'a geração em monoterapia na terapêutica sintomática do resfriado comum poderá estar associado a um risco acrescido de efeitos sedativos e gastrointestinais, com uma menor força de recomendação (SOR B). Este aspecto é relevante para os pais dos pacientes pediátricos que poderão confundir esta sedação com uma prostração que é um sinal de alarme num contexto infeccioso.

Estas conclusões actuais confirmam, de resto, a suspeita antiga de ineficácia dos AHs. De facto, os estudos que documentam a ausência de efectividade dos anti-histamínicos em monoterapia são suportados por ensaios de fisiologia humana envolvendo indivíduos com resfriado comum. Estes últimos revelaram que a histamina, os mastócitos ou os basófilos (envolvidos na rinite alérgica) não têm qualquer papel na rinite infecciosa, enquanto que os níveis de cininas estão relacionados com a severidade dos sintomas, parecendo ser estas os mediadores inflamátorios protagonistas neste processo infeccioso. ${ }^{13}$
Entretanto, poderá ser iniciado um processo especulativo em torno da questão: porque tem vindo o médico a acreditar na efectividade dos AHs no resfriado comum durante a sua prática clínica? Três razões possíveis podem ser desenhadas.

Primeira, certamente o médico reconhecerá que, perante um resfriado comum, frequentemente prescreve concomitantemente um AINE. Os AINEs têm razoável grau de evidência a suportar o seu uso e poderão, então, ser responsáveis pelo benefício clínico. ${ }^{14}$

A segunda razão prende-se com o facto de alguns doentes com sintomatologia respiratória superior não apresentarem um resfriado comum mas, sim, uma agudização da sua rinite alérgica (por vezes, não-diagnosticada). O benefício do anti-histamínico emerge por actuar, portanto, em contexto alérgico e não em campo infeccioso.

Finalmente, a própria história natural do resfriado comum poderá conduzir o médico prescritor até uma ideia errada. De facto, depois de um pico sintomático nos terceiro e quarto dias de evolução, os sintomas do resfriado melhoram consideravelmente. ${ }^{3}$ É, frequentemente, nestes dias 3 e 4 que o doente procura o seu médico e lhe é prescrito o anti-histamínico. Na realidade, a melhoria sintomática poderá dever-se simplesmente à previsível história natural da maioria dos resfriados comuns e não à toma do $\mathrm{AH}$ que entretanto inicia.

Em conclusão, os anti-histamínicos em monoterapia não têm benefício clínico no tratamento sintomático do resfriado comum e poderão estar associados a um risco acrescido de efeitos laterais. De maneira que, em matéria de evidência, o uso de antihistamínicos no resfriado comum é apenas evidentemente comum.

\section{AGRADECIMENTOS}

À Dra. Raquel Braga, à Dra. Mónica Granja e ao Dr. Luís Filipe Cavadas, pela amizade e pelo constante suporte científico e crítico.

\section{REFERÊNCIAS BIBLIOGRÁFICAS}

1. Simasek M, Blandino DA. Treatment of the common cold. Am Fam Phys 2007 Feb 15; 75 (4): 515-20.

2. Sutter Al, Lemiengre M, Campbell H, Mackinnon HF. Antihistamines for the common cold. Cochrane Database Syst Rev 2003;(3):CD001267.

3. Eccles R. Undestanding the symptoms of the common cold and influenza. Lancet Infect Dis 2005 Nov; 5 (11): 718-25.

4. Sung V, Cranswick N. Cough and cold remedies for children. Aust Prescr 2009; 32 (5): 122-4.

5. Del Cuvillo A, Sastre J, Montoro J, Jáuregui I, Ferrer M, Dávila I, et al. Use of 
antihistamines in pediatrics. J InvestigAllergol Clin Immunol 2007;17 Suppl 2: 28-40.

6. Gaffey MJ, Kaiser DL, Hayden FG. Ineffectiveness of oral terfenadine in natural colds: evidence against histamine as a mediator of common cold symptoms. Pediatr Infect Dis J 1988 Mar; 7 (3): 223-8.

7. Ebell MH, Siwek J, Weiss BD, Woolf SH, Susman J, Ewingman B, et al. Strength of Recommendation Taxonomy (SORT): a patient-centered approach to grading evidence in the medical literature. Am Fam Phys 2004 Feb 1;69 (3): 548-56.

8. Irwin RS, Baumann MH, Bolser DC, Boulet LP, Braman SS, Brightling CE, et al. Diagnosis and management of cough executive summary: ACCP evidence-based clinical practice guidelines. Chest 2006 Jan; 129 (1 Suppl): 1S23s.

9. Anderson G,Avery W, Cunningham B, Graft D, Hunteman T, Jenkins J, et al. Diagnosis and Treatment of Respiratory Illness in Children and Adults. Institute of Clinical Systems Improvement; 2008. Disponível em: http://www.icsi.org/respiratory_illness_in_children_and_adults_guideline_13116.html [acedido em 02/02/2012].

10. Berkowitz RB, Tinkelman DG. Evaluation of oral terfenadine for treatment of the common col. Ann Allergy 1991 Dec; 67 (6): 593-7.

11. Sakchainanont B, Ruangkanchanasetr S, Chantarojanasiri T, Tapasart C, Suwanjutha S. Effectiveness of antihistamines in common cold. Med Assoc Thai 1990 Feb; 73 (2): 96-101.
12. Paul IM, Yoder KE, Crowell KR, Shaffer ML, MCMillan HS, Carlson LC, et al. Effect of dextromethorphan, diphenhydramine, and placebo on nocturnal cough and sleep quality for coughing children and their parents. Pediatrics 2004 Jul; 114 (1): e85-e90.

13. Naclerio RM, Proud D, Kagey-Sobotka A, Lichtenstein LM, Hendley JO, Gwaltney JM Jr. Is histamine responsible for the symptoms of rhinovirus colds? A look at the inflammatory mediators following infection. Pediatr Infect Dis J 1988 Mar; 7 (3): 218-22.

14. Kim SY, Chang YJ, Cho HM, Hwang YW, Moon YS. Non-steroidal anti-inflammatory drugs for the common cold. Cochrane Database Syst Rev 2009 Jul 8; (3): CD006362.

\section{CONFLITOS DE INTERESSE}

O autor declara não possuir qualquer conflito de interesse.

\author{
ENDEREÇO PARA CORRESPONDÊNCIA \\ José Agostinho Santos \\ Rua Hernâni Torres, $73,4^{\circ}$ Dir \\ 4200 - 320 Porto \\ E-mail: zeagostinho@hotmail.com
}

Recebido em 14/07/2011

Aceite para publicação em 02/12/2011

\section{ABSTRACT}

\section{ANTIHISTAMINES IN THE COMMON COLD: A COMMON PRESCRIPTON FOR A COMMON CONDITION}

Goals: Antihistamines are popular among doctors for treatment of symptoms of the common cold. The aim of this paper is to review the evidence for the effectiveness and safety of antihistamines as the sole treatment for symptoms of the common cold in children and adults.

Data sources: Medline, evidence-based medicine internet sites, Index of Portuguese Medical Journals and references of selected articles.

Review methods: Clinical guidelines, systematic reviews, and randomized controlled trials (RCT), published between January 1980 and October 2010 were collected using the MeSH terms: antihistamine and common cold. The Strength Of Recommendation Taxonomy (SORT) scale of the American Academy of Family Physicians was used for assigning levels of evidence and the strength of recommendation.

Results: Seventy articles were found and seven were selected for this review. These include two guidelines on the management of the common cold that do not recommend antihistamines as a sole treatment option and one Cochrane meta-analysis that found no clinical benefit from the use of antihistamines alone. The results of some studies included suggested an increased risk of sedative effects with the use of first generation antihistamines. The authors of this meta-analysis recommend discontinuing the practice of prescribing these drugs in the common cold (level of evidence 1). Four RCT found that different antihistamines were no more effective than placebo in reducing acute cough. Three studies showed no significant differences in side effects (level of evidence 1).

Conclusions: The evidence shows that the use of antihistamines as monotherapy in symptomatic treatment of the common cold is ineffective in children and adults (SOR A). Many trials suggest an increased risk of sedative effects with this therapy (SOR B). Sedation may be confused with prostration that would suggest a worsening of the clinical situation. This may add an unnecessary cost to a common prescription.

Keywords: Antihistamine; Common Cold. 